\title{
Morfología de las piezas bucales y hábitos alimenticios de Leptonema y Smicridea (Trichoptera: Hydropsychidae) del río Gaira, Sierra Nevada de Santa Marta, Colombia
}

\author{
Kevin Daniel Álvarez-Soraca*, Cesar Enrique Tamaris-Turizo \& Cristian José Guzmán-Soto \\ Grupo de Investigación en Biodiversidad y Ecología Aplicada, Universidad del Magdalena, Carrera 32 No. $22-08$, \\ Santa Marta, Colombia; kedaalso07@gmail.com, ctamaris@unimagdalena.edu.co,guzmansotocj@gmail.com \\ * Correspondencia
}

Recibido 21-X-2016. Corregido 09-V-2017. Aceptado 12-VI-2017.

\begin{abstract}
Morphology of mouthparts and feeding habits of Leptonema and Smicridea (Trichoptera: Hydropsychidae) from Gaira river, Sierra Nevada de Santa Marta, Colombia. The genera Leptonema and Smicridea belong to the family Hidropsychidae and constitute one of the most important groups in the nutrient dynamics in the tropical aquatic ecosystems, which diversity of microhabitats is exploited by their larvae. However, there is lack of information concerning the detailed and comparative descriptions of mouthparts between these two genera, and their possible relation with their diet. The aim of this paper was to describe the morphology of the mouthparts and the feeding habits of both genera, in the middle basin of the Gaira River (Sierra Nevada de Santa Marta, Colombia). The samples were collected on a monthly basis, with a surber net, between October and December of 2014, and May of 2015. A total of 15 larvae per genus were taken and the Blue Zen Software was used to determine the morphometric measurements of the cephalic pieces. Measurements were taken on photographs obtained from an AxioCam ERc5s camera adapted to a Nikon SMZ $745 \mathrm{~T}$ stereoscope. To describe the mouthparts a M205A microscope-stereoscope Leica was used. The dietary habits of 15 individuals in total were evaluated by means of the analysis of stomach contents. A discriminant analysis of Hotelling was applied to the morphometric measurements. There were significant differences in head area (HA), head width (HW3), head length (HL2) and left mandible length (LML). In the morphological descriptions were found differences in: head, labrum and submentum. Dietaries profiles were performed to the stomach contents for both genera and the items with highest percentage for Leptonema was MOPG and MOPF with $38 \%$ and $32 \%$, respectively, and for Smicridea MOPF (38\%) and MOPG (34\%) with significant differences $(\mathrm{F}=8.8298, \mathrm{p}<0.05)$. This study indicated that the evaluated individuals consume a wide variety of resources and the difference of the diet of both genera can be related to the detected morphological differences. Rev. Biol. Trop. 65 (4): 1231-1244. Epub 2017 December 01.
\end{abstract}

Key words: aquatic insects, morphometric, gut contents, mouthparts, food item.

Hydropsychidae es una familia abundante y de una amplia distribución geográfica dentro del orden Trichoptera, la cual tiene cerca de 1500 especies descritas a nivel mundial (Valladolid, Martínez, \& Arauzo, 2007; Nessimian \& Dumas, 2010). Pueden ser reconocidas por la presencia de branquias abdominales ramificadas y un cuerpo recubierto de setas (Springer, Serrano, \& Zepeda, 2010). Los géneros más comunes de esta familia son Leptonema
(Macronematinae) y Smicridea (Smicrideinae) con 70 y 109 especies, respectivamente, descritas en Sudamérica, donde ambos géneros se encuentran ampliamente distribuidos (Angrisano \& Sganga, 2009). Leptonema se caracteriza por presentar en ocasiones un color verde intenso y por ser de gran tamaño entre los tricópteros (Posada \& Roldán, 2003), en estado larval construyen redes finas con materiales orgánicos y minerales para filtrar el agua 
(Merritt, Cummnins, \& Berg, 2008). Mientras que las larvas de Smicridea construyen refugios fijos, generalmente con fragmentos de plantas y arena ubicados en las grietas de las piedras (Duque, 2009). Regularmente se localizan en zonas de corrientes rápidas, sustratos rocosos y espacios abiertos con mayor incidencia de luz solar (Pes, 2005).

Algunos aspectos ecológicos de la familia Hydropsychidae en los sistemas lóticos, están relacionados con su elevada densidad y biomasa; además, juegan un papel importante en los eslabones de las redes tróficas y las larvas son parte de la dieta de aves, peces y ranas (Wiggins, 2004; Huamantinco \& Nessimian, 2000; Angrisano \& Korob, 2001). Por otro lado, se considera que estos organismos cumplen funciones tróficas diferenciales, por lo cual han sido asignados a diversos grupos funcionales como colector-filtrador o fragmentadores (Chará, Chará, Zúñiga, Pearson, \& Boyero, 2012; Granados, 2013; Guzmán-Soto \& Tamaris-Turizo, 2014). No obstante, basados en estudios de contenidos estomacales, el género Smicridea pareciera tener un rol depredador de invertebrados (Reynaga, 2009; Rodríguez, Gracia, \& Peña, 2014).

Los trabajos que han evaluado aspectos morfológicos relacionados con las piezas bucales de Leptonema y Smicridea concluyen que es importante incluir dichos análisis para generar claves taxonómicas; o bien, incorporar algunos temas de la biología de los organismos (Nessimian \& Dumas, 2010). Dentro de los trabajos que abordan estos géneros se destacan los desarrollados por Muñoz (1999) quien enfatizó en la descripción e ilustración de una nueva especie del genero Leptonema en Costa Rica, elaborando una clave taxonómica para la identificación de los machos de dicha especies. Mientras que en Colombia, se destaca el trabajo realizado por Posada y Roldán (2003) basado en la elaboración de claves ilustradas y la diversidad de las larvas de Trichoptera en el Nor-Occidente del país.

A pesar que se conocen aspectos sobre la ecología y la distribución de esta familia en Colombia, no existen descripciones comparativas sobre la morfología del aparato bucal y piezas cefálicas de los géneros Leptonema y Smicridea, y su posible relación con sus dietas. Por lo anterior, se busca describir la morfología de los aparatos bucales con detalle de sus piezas, así como la dieta de éstos géneros. Teniendo en cuenta que existen algunas diferencias morfológicas en las branquias y la forma de la cabeza, se espera que estos géneros difieran también en la morfología de sus piezas bucales y en sus dietas.

\section{MATERIALES Y MÉTODOS}

Área de estudio: Los organismos evaluados se recolectaron en el río Gaira, cuya corriente de agua tiene una longitud aproximada de $32.53 \mathrm{~km}$ desde su nacimiento hasta su desembocadura (Frayter, Pabón, \& Valero, 2000). El río está ubicado en el departamento del Magdalena, Colombia. Las muestras fueron recolectadas en la cuenca media a una altura de $900 \mathrm{msnm}$, entre los $11^{\circ} 07^{\prime} 47.8^{\prime \prime}$ $\mathrm{N}-74^{\circ} 05^{\prime} 42.04$ " W, (cuyo sector hace parte de la hacienda La Victoria). El sitio presenta una formación vegetal de bosque húmedo subtropical. En el lecho del río predominan rocas y gravas, propiciando zonas de corrientes rápidas, aunque se puede observar zonas de corriente lenta, lo que genera diversos microhábitats (Guerrero, Manjarrés, \& Núñez, 2003; Guerrero \& Sarmiento, 2010; Tamaris-Turizo, Rodríguez-Barrios, \& Ospina-Torres, 2013).

\section{Procesamiento del material biológico:}

Se realizaron tres muestreos cubriendo los periodos de lluvias (octubre, noviembre e inicios de diciembre 2014) y sequía (finales de diciembre 2014 y junio 2015). En la cuenca media del río se seleccionó un tramo de $50 \mathrm{~m}$ en el que se tomaron cinco muestras, mediante una red Surber (apertura de malla de $250 \mu \mathrm{m}$ ) durante 5 minutos, removiendo manualmente el sustrato frente a la red en contra de la corriente (Darrigran, Vilches, Legarralde, \& Damborenea, 2007). El material extraído fue almacenado en bolsas plásticas y preservado en etanol al $96 \%$, luego se transportaron 
al laboratorio para su posterior análisis. Los organismos se separaron usando un estereoscopio (Nikon SMZ 745T) y las larvas se identificaron hasta el nivel de género utilizando las claves taxonómicas y guías propuestas por Posada y Roldán (2003) y Wiggins (2004).

Descripción del aparato bucal: Para la descripción, se utilizó 15 individuos en estado larval de ambos géneros, usando un microscopio-estereoscopio Leica M205A, y para la estimación morfométrica, se implementó una cámara fotográfica AxioCam ERc5s acoplada a un estereoscopio Nikon SMZ 745T. Con la ayuda del Software Zen Blue se calcularon las siguientes medidas: longitud total del cuerpo, área, ancho y largo de cada una de las piezas bucales de los individuos (Cuadro 1). Posteriormente, se realizó el montaje del aparato bucal ordenando cada pieza de acuerdo a su posición original con el fin de observar labrum, mandíbulas y labio maxilar compuesto por dos palpos (Fig. 1). Finalmente, se procedió a fotografiar cada una de las piezas en un microscopio-estereoscopio Leica M205A con cámara integrada Leica DFC450.

Análisis del contenido estomacal: Se empleó la técnica propuesta por Tomanova, Goitia y Helesic (2006), la cual consiste en extraer el tracto de uno a diez individuos de cada taxón (de acuerdo a su abundancia y con contenido en su interior). Del género Leptonema se evaluaron 8 individuos y Smicridia 7, para un total de 15 en estadio larval. El contenido estomacal fue homogeneizado individualmente sobre una lámina portaobjeto con glicerina; seguidamente, se examinaron y fotografiaron 20 campos de manera aleatoria, mediante una cámara ajustada a un microscopio ZEISS Primo Star en diferentes aumentos. Posteriormente, con ayuda del software Zen Blue, se delimitó el área de cada ítem en los campos fotografiados, para describir y cuantificar los porcentajes de estos. Las categorías alimentarias que se consideraron fueron: Tejido

CUADRO 1

Variables medidas en las diferentes piezas bucales de Leptonema y Smicridea, con sus respectivos códigos

TABLE 1

Variables measured in the different buccal parts of Leptonema and Smicridea, with their respective code

\begin{tabular}{|c|c|c|c|c|c|}
\hline \multicolumn{2}{|c|}{ Variable } & \multirow{2}{*}{$\begin{array}{c}\text { Código } \\
\text { AC }\end{array}$} & \multicolumn{2}{|c|}{ Variable } & \multirow{2}{*}{$\begin{array}{c}\text { Código } \\
\text { AMI }\end{array}$} \\
\hline Cabeza & Área & & Mandíbula izquierda & Area & \\
\hline & Ancho1 & ANC1 & & Largo & LRMI \\
\hline & Ancho2 & ANC2 & & Ancho & ANMI \\
\hline & Ancho3 & ANC3 & & Lado1 & LMI1 \\
\hline & Largo1 & LC1 & & Lado2 & LMI2 \\
\hline & Largo2 & $\mathrm{LC} 2$ & & Lado3 & LMI3 \\
\hline & Largo3 & LC3 & Mandíbula derecha & Área & AMD \\
\hline \multirow[t]{5}{*}{ Labrum } & Área & $\mathrm{AL}$ & & Largo & LRMD \\
\hline & Largo & LL & & Ancho & ANMD \\
\hline & Ancho1 & ANL1 & & Lado1 & LMD1 \\
\hline & Ancho2 & ANL2 & & Lado2 & LMD2 \\
\hline & Ancho3 & ANL3 & & Lado3 & LMD3 \\
\hline \multirow[t]{3}{*}{ Labrum inferior 1} & Área & ALI1 & Labrum inferior medio & Área & ALIM \\
\hline & Largo & LRLI1 & & Base & BLIM \\
\hline & Base & BLI1 & & Largo & LRLIM \\
\hline \multirow[t]{3}{*}{ Labrum inferior 2} & Área & ALI2 & & & \\
\hline & Largo & LRLI2 & & & \\
\hline & Base & BLI2 & & & \\
\hline
\end{tabular}



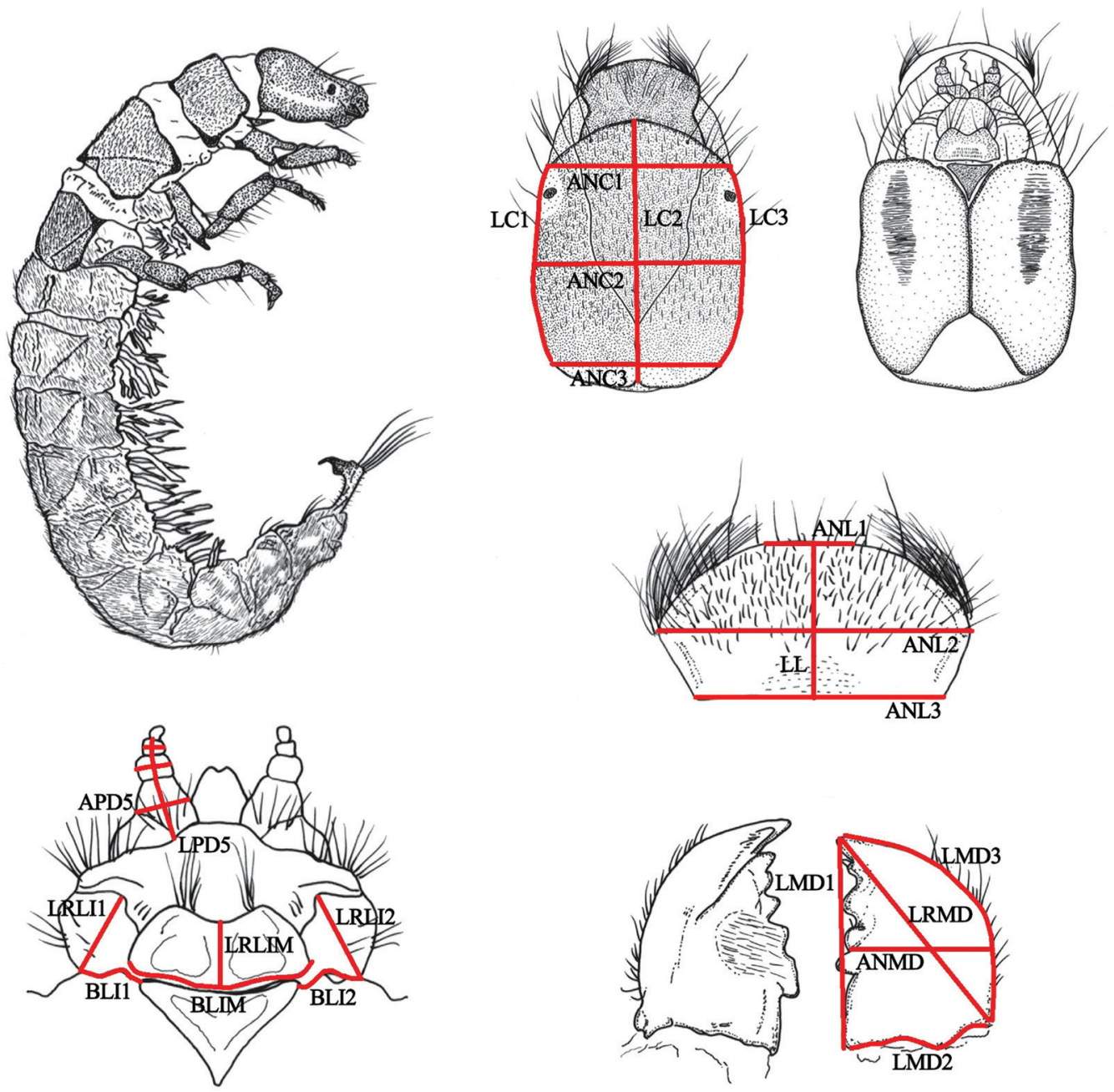

Fig. 1. Bosquejo de las medidas tomadas a las piezas bucales con sus respectivos acrónimos. Modificado de Nessimian \& Dumas (2010).

Fig. 1. Illustration of the mouthparts measured with their acronyms. Modified from Nessimian \& Dumas, (2010).

de restos animales (RA), Tejido de plantas vasculares (TPV), Microalgas (MCR), Hongos (HN), Materia Orgánica Particulada Gruesa, > $1 \mathrm{~mm}(\mathrm{MOPG})$ y Materia Orgánica Particulada Fina, $50 \mu \mathrm{m}-1 \mathrm{~mm}$ (MOPF). La clasificación de la MOPG y la MOPF se tomaron de Vannote, Minshall, Cummins, Sedell y Cushing (1980).

Tratamiento de datos: Las medidas de las piezas cefálicas se estandarizaron dividiendo cada variable entre la longitud total (LT), para obtener una proporción que pudiera ser comparable entre los organismos. Para conocer las posibles diferencias entre las métricas de los dos géneros, inicialmente se seleccionaron las métricas con mejor correlaciones evidenciadas en un Análisis de Componentes Principales (ACP); posteriormente, se realizó un Análisis Discriminante de Hotelling. Se realizaron comparaciones bivariadas entre las métricas de los dos géneros, mediante las pruebas t-test y U-Mann Whitney, previa 
comprobación de la normalidad de los datos. Finalmente, se compararon las dietas entre los géneros, mediante un Análisis Discriminante de Hotelling. Todos los análisis fueron realizados en el programa estadístico $\mathrm{R}$ 2.14.2 Patched (R Development Core Team, 2012).

\section{RESULTADOS}

Los organismos del genero Leptonema (Fig. 2A) presentaron una longitud promedio de $26.4( \pm 0.49 \mathrm{~mm})$, mientras que Smicridea (Fig. 3A) un promedio de $16.1( \pm 0.22$ $\mathrm{mm})$. Se evidenció que existen diferencias significativas en la LT entre ambos géneros $(\mathrm{t}=18.99, \mathrm{p}<0.01)$.

Leptonema - Cabeza: Coloración café oscura, periocular claro, esclerotizada, cubierta de setas cortas. Área promedio de $5.8( \pm 0.09$ $\mathrm{mm}^{2}$ ). Ligeramente más ancha en la parte posterior $2.3( \pm 0.02 \mathrm{~mm})$ que en la anterior

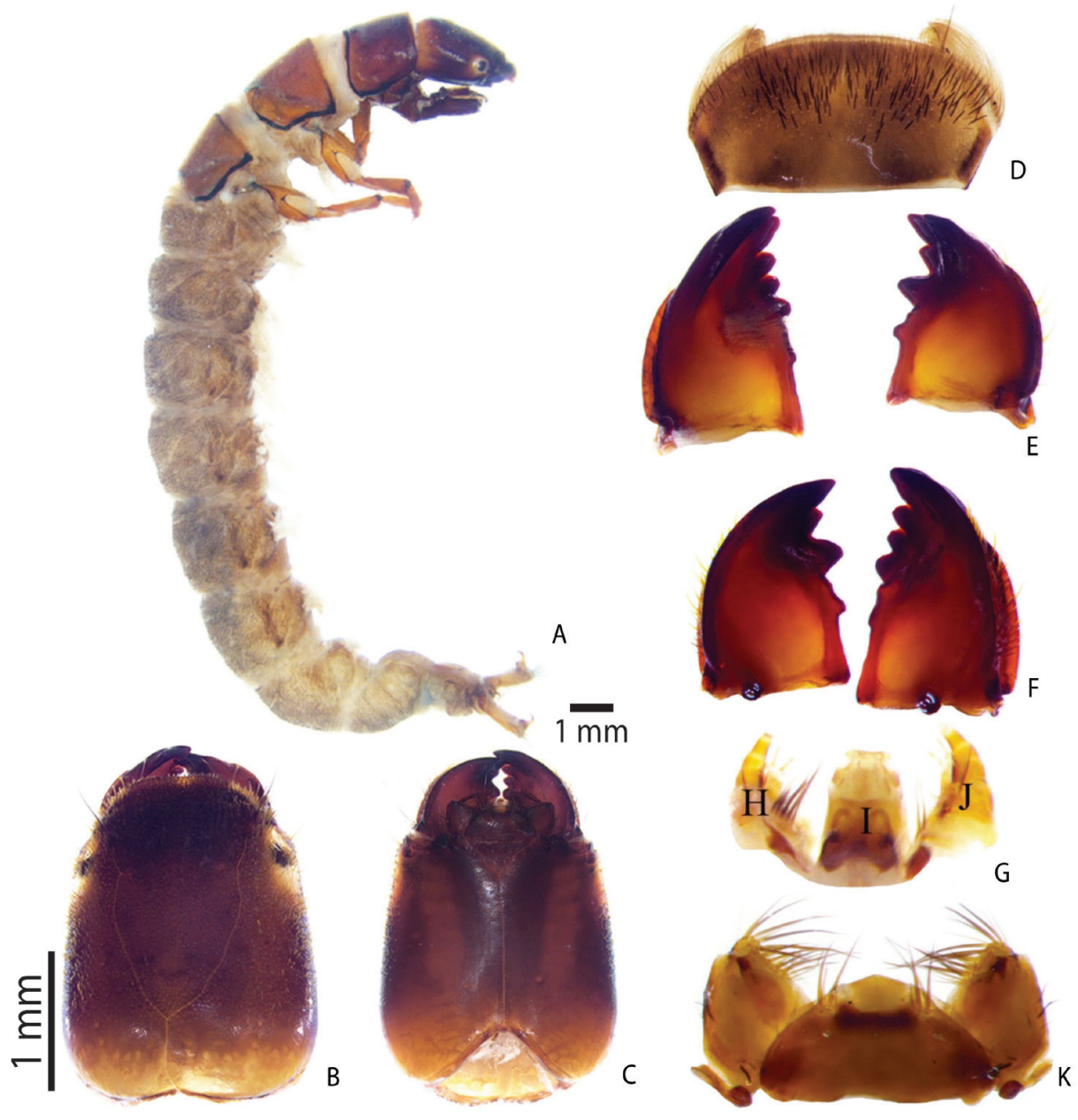

Fig. 2. Larva de Leptonema (Trichoptera: Hydropsychidae): A) vista lateral del cuerpo. B) cabeza, vista dorsal. C) cabeza, vista ventral. D) labro, vista dorsal. E) mandíbulas, vista dorsal. F) mandíbulas, vista ventral. G) complejo hipofaringeal, vista ventral. H) palpo derecho. I) faringe. J) palpo izquierdo. K) submentón, vista ventral.

Fig. 2. Leptonema larvae (Trichoptera: Hydropsychidae): A) side view of the body. B) head, dorsal view. C) head, ventral view. D) labrum, dorsal view. E) mandible, dorsal view. F) mandible, ventral view. G) hipofaringeal complex, ventral view, H) palpi right. I) pharynx. J) left palpi. K) submentón, ventral view. 

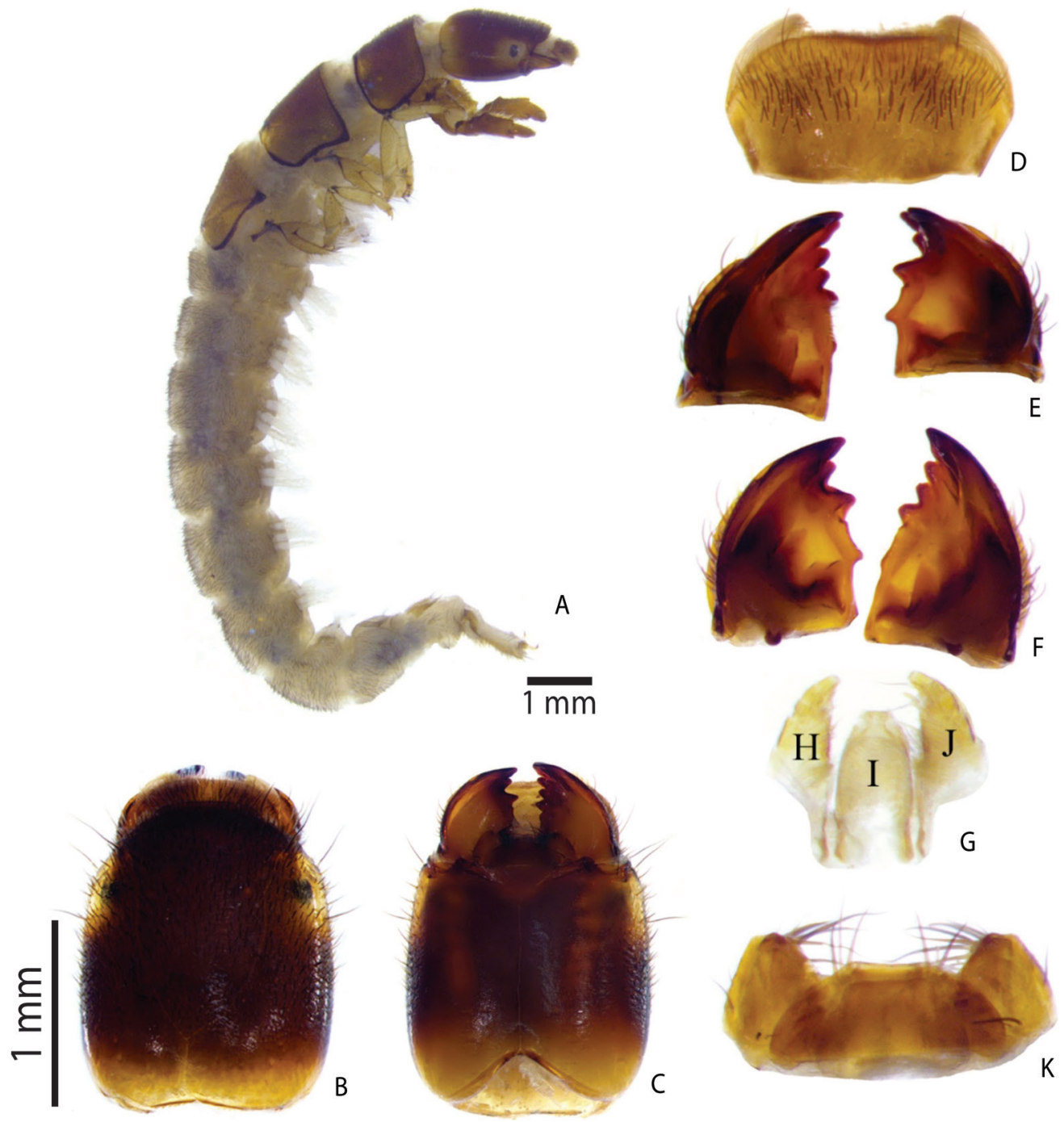

Fig. 3. Larva de Smicridea (Trichoptera: Hydropsychidae): A) vista del cuerpo. B) cabeza, vista dorsal. C) cabeza, vista ventral. D) labro, vista dorsal. E) mandíbulas, vista dorsal. F) mandíbulas, vista ventral. G) Complejo hipofaringeal, vista ventral. H) palpo derecho. I) faringe. J) palpo izquierdo. K) submentón, vista ventral.

Fig. 3. Smicridea larvae (Trichoptera: Hydropsychidae): A) habitus view. B) head, dorsal view. C) head, ventral view. D) labrum, dorsal view. E) mandible, dorsal view. F) mandible, ventral view. G) hipofaringeal complex, ventral view. H) palpi right. I) pharynx. J) left palpi. K) submentón, ventral view.

$1.9( \pm 0.04 \mathrm{~mm})$, antenas pequeñas. En vista dorsal (Fig. 2B): sutura coronal corta tenue en el margen posterior, margen de la sutura frontoclipeal triangular. Apotema frontoclipeal en forma de "V" alargado, margen anterior recto de forma irregular y puntiagudo posteriormente. En el margen anterior, presencia de setas de diferentes tamaños, no más grandes que las antenas. En vista ventral (Fig. 2C): ausencia de setas. Estructura de estridulación larga que se extiende desde el borde anterior hasta el borde posterior, con rayas finas bien marcadas. Margen posterior redondeado con una hendidura mesial, sutura ecdisial divide completamente 
la gena. Apotema posteroventral esclerotizado, triangular y diminuto, presencia de máculas. El margen anterior convexo y aserrado. Submentón anteroventral esclerotizado y semicircular. Apotema anteroventral esclerotizado, con forma triangular.

Labrum: Presenta un color café claro, área promedio de $0.7\left( \pm 0.01 \mathrm{~mm}^{2}\right)$, más ancho que largo, con los ángulos anteriores redondeados, está cubierto en la mayoría de su superficie con setas cortas de color negro, a excepción de la base de este. Vista dorsal: margen anterior semiovalado, con un ancho de 0.7 ( $\pm 0.04 \mathrm{~mm})$, región media más ancha con un promedio 1.2 $( \pm 0.01 \mathrm{~mm})$ y base del margen posterior con promedio de $0.9( \pm 0.02 \mathrm{~mm})$. Margen anterior con setas cortas, presenta un par de setas medianas y delgadas en el margen central. Setas largas formando un mechón en el margen lateral de ambos lados (Fig. 2D). Vista ventral: setas delgadas largas y curvadas hacia la región media, que inician desde el borde anterior, las cuales tienen como función no dejar salir las partículas de alimento. En algunos individuos el labrum se encontró retraído o extendido, se puede considerar que el labrum tiene un movimiento retráctil y tiene como función proteger el complejo hipofaringeal.

Mandíbulas: Completamente asimétricas y fuertemente esclerotizadas. El margen cóncavo interno de las mandíbulas forma dos bordes cortantes (superior e inferior), función cortar el alimento (Fig. 2E y Fig. 2F). Mandíbula izquierda: En vista dorsal, con un área promedio de $0.5\left( \pm 0.04 \mathrm{~mm}^{2}\right)$, la región con la longitud más larga con promedio de 1.0 ( \pm $\left.0.05 \mathrm{~mm}^{2}\right)$ y la parte más ancha con $0.5( \pm$ $0.04 \mathrm{~mm}^{2}$ ), con ocho dientes de forma irregular, un diente dorsal apical, con dos dientes adicionales dorso apical (uno encima del otro), seguido de tres dientes en el área incisiva los cuales varían en formas y tamaño (triangulares o redondeados), en el área molar dos dientes fusionados. Presencia de un penacho de setas medianas cerca al área dorsomolar. Mandíbula derecha: En vista dorsal, con un área promedio de $0.4\left( \pm 0.03 \mathrm{~mm}^{2}\right)$, más corta que la mandíbula izquierda, la región con la longitud más larga con promedio de $0.9( \pm 0.05 \mathrm{~mm})$ y la parte más ancha con un promedio $0.5( \pm 0.04 \mathrm{~mm})$. Con seis dientes irregulares, un diente dorsal apical, con un diente adicional (uno encima del otro), seguido de dos dientes en el área incisiva que varían en formas y tamaño (triangulares o redondeados) y en la región molar dos dientes fusionados con forma redondeada. Ausencia de setas en la región dorsomolar. Presencia de setas cortas oscuras en el margen externo de ambas mandíbulas.

Complejo hipofaringeal: Parcialmente esclerotizado (donde se abre el tubo digestivo Fig. 2I) ubicado ventralmente debajo de las mandíbulas (Fig. 2G). Maxila con palpos y galea bien diferenciados. Presencia de dos palpos maxilares en posición lateral (Fig. $2 \mathrm{H}$ y Fig. 2J), compuestos por cinco artejos, el segundo dos veces más ancho que el primero y así sucesivamente, cumplen un papel importante al llevar la comida hacia la faringe, gracias a su capacidad retráctil que le permite sacar sus palpos más allá de sus mandíbulas. Galea en forma de pequeñas espinas que se proyecta de los palpos maxilares. Submentón (labio) en vista ventral: esclerotizado, puntiagudo en el margen anterior, con muchas setas largas, un área promedio de $0.2\left( \pm 0.01 \mathrm{~mm}^{2}\right)$, longitud de la base promedio de $0.7( \pm 0.01 \mathrm{~mm})$ y en la región media largo promedio de $0.4( \pm$ $0.01 \mathrm{~mm}$ ), con capacidad de movimiento, el cual le permite cerrarse y proteger el complejo hipofaringeal (Fig. 2K). Submentón y maxila están fuertemente fusionados (formando un labio maxilar), con setas de distintas longitudes y grosores.

Smicridea - Cabeza: Coloración café oscuro, esclerotizada, levemente rectangular. Área promedio de $2.5\left( \pm 0.04 \mathrm{~mm}^{2}\right)$, recubierta de setas cortas y finas en la región dorsal, antenas diminutas. En vista dorsal (Fig. 3B): margen anterior con un ancho promedio de $1.2( \pm 0.02 \mathrm{~mm})$, región media y posterior con un ancho promedio de $1.5( \pm 0.02 \mathrm{~mm})$. 
Sutura coronal corta tenue en la región posterior, sutura frontoclipeal en forma triangular tenue, Apotema frontoclipeal en forma de "V" en el margen anterior, irregular y puntiagudo en la región posterior. Presencia de setas en el margen anterior de distintos tamaños, no más grandes que las antenas. En vista ventral (Fig. 3C): ausencia de setas, estructura de estridulación que se extiende desde el borde anterior hasta el posterior, con rayas finas de manera horizontal. Sutura ecdisial que divide completamente la gena, margen posterior redondeado con una hendidura mesial, apotema posteroventral diminuto, esclerotizado y triangular, presencia de máculas. Margen de la región anterior de forma convexa y aserrado. Apotema anteroventral esclerotizado, con forma triangular. Submentón esclerotizado, semicircular anteroventral.

Labrum: Transversal de color café claro, área promedio de $0.3\left( \pm 0.01 \mathrm{~mm}^{2}\right)$, más ancho que largo, con los ángulos anteriores redondeados, recubierto en la mayoría de su superficie con setas de color negro. Vista dorsal: margen anterior recto diferente de Leptonema, ancho promedio de $0.6( \pm 0.02 \mathrm{~mm})$, región media más ancha con $0.8( \pm 0.02 \mathrm{~mm})$ y margen posterior más angosto $0.4( \pm 0.01 \mathrm{~mm})$. Margen anterior con setas cortas y delgadas, un par de setas finas medianas en el margen central, separadas una de la otra. Margen anterolaterales con setas largas formando un mechón de ambos lados (Fig. 3D). Vista ventral: setas delgadas largas y curvadas hacia la región media, que van formando una hilera desde el borde anterior al posterior tienen como función minimizar la pérdida del alimento. En algunos individuos el labrum se observó retraído y en otros extendido, se puede considerar que el labrum tiene un movimiento retráctil, al igual que en Leptonema, y cumple la misma función.

Mandíbulas: Asimétricas, bien desarrolladas y fuertemente esclerotizadas. El margen cóncavo interno de las mandíbulas forma dos bordes cortantes (superior e inferior) (Fig. 3E, Fig. 3F). Mandíbula izquierda: en vista dorsal con un área promedio de $0.3\left( \pm 0.01 \mathrm{~mm}^{2}\right)$, región con la longitud más larga con promedio de $0.7( \pm 0.02 \mathrm{~mm})$ y la parte más ancha $0.4( \pm$ $0.02 \mathrm{~mm}$ ). Presencia de ocho dientes de forma irregular, un diente dorsal apical, se presentan dos dientes adicionales sobre el diente dorso apical, seguido de tres dientes en el área incisiva, estos varían en formas y tamaño (triangulares o redondeados). En la región molar dos dientes fusionados. Presencia de un penacho de setas medianas finas cerca de la región dorsomolar. Mandíbula derecha: en vista dorsal con un área promedio de $0.2\left( \pm 0.01 \mathrm{~mm}^{2}\right)$, región con la longitud más larga con promedio de $0.6( \pm 0.01 \mathrm{~mm})$ y la parte más ancha con un promedio de $0.3( \pm 0.01 \mathrm{~mm})$. Con seis dientes irregulares, un diente en posición dorsoapical, presencia de un diente adicional sobre el diente dorso apical, seguido de dos dientes en el área incisiva que varían tanto de forma y tamaño. En la región molar dos dientes fusionados, el primero redondeado y el segundo en forma puntiaguda. Ausencia de setas en la región dorsomolar y presencia de setas oscuras cortas en el margen externo de ambas mandíbulas.

Complejo hipofaringeal: Sin esclerotizar (donde se abre el tubo digestivo Fig. 3I) ubicado ventralmente debajo de las mandíbulas (Fig. $3 \mathrm{G})$. Maxila con palpos y galea bien diferenciados. Presencia de dos palpos maxilares en posición lateral (Fig. $3 \mathrm{H}$ y Fig. 3J), compuestos por cinco artejos, el segundo dos veces más ancho que el primero y así sucesivamente, cumplen un papel importante al llevar la comida hacia la faringe, gracias a su capacidad retráctil que le permite sacar sus palpos más allá de sus mandíbulas. Galea en forma de pequeñas espinas que surgen de los palpos maxilares. Submentón (labio) en vista ventral: esclerotizado parcialmente, margen anterior recto diferente a Leptonema, con pocas setas largas, un área promedio de $0.1\left( \pm 0.01 \mathrm{~mm}^{2}\right)$, longitud de la base promedio de $0.5( \pm 0.01 \mathrm{~mm})$ y en la región media con un largo promedio de 0.2 ( \pm $0.01 \mathrm{~mm}$ ), con capacidad de movimiento, el cual le permite cerrarse y proteger el complejo hipofaringeal (Fig. 3K). Submentón y maxila 
están fuertemente fusionados (formando un labio maxilar), con setas de distintos tamaños y grosores.

\section{Comparación morfológica entre Lepto-} nema y Smicridea: El análisis de componentes principales en el primer eje explicó un $66.9 \%$ de la varianza y el segundo un $18.1 \%$. Este ACP permitió detectar las métricas (variables) que aportan mayormente a la variación de los datos: área de la cabeza (AC), ancho de la cabeza 3 (ANC3), largo de la cabeza 2 (LC2), largo de la mandíbula izquierda 1 (LMI1), largo de la mandíbula izquierda 3 (LMI3), largo de la mandíbula derecha (LRMD), ancho de la mandíbula derecha (ANMD), lado de la mandíbula derecha 1 (LMD1), lado de la mandíbula derecha 3 (LMD3), largo del labrum inferior 1(LRLI1), largo del labrum inferior medio (LRLIM). Dichas variables se usaron en la prueba de Hotelling, la cual corroboró las diferencias significativas entre los géneros $(\mathrm{F}=81.775, \mathrm{p}<0.05)$. En la figura 4 se muestra un diagrama de caja y bigote donde se comparan las métricas cefálicas.

La variable AC presentó diferencias significativas entre ambos géneros (t-test $=16.08$, $\mathrm{p}<0.05$ ); al igual que LC2, LMI1, LRMD, LMD1, LRLIM (U-Mann Withney, $\mathrm{p}<0.05$ ). Mientras que ANC3, LMI3, ANMD, LMD3, LRLI1 no evidenciaron diferencias entre los géneros (U-Mann Withney, p>0.05).

Dietas: En general, la dieta de ambos géneros se caracterizó por estar compuesta mayoritariamente por MOPG, seguida de
MOPF y finalmente TPV. Los RA, las MCR y los HN se observaron en una menor proporción (Cuadro 2). En Leptonema se encontró MOPF, MOPG y TPV, con $32 \%$, $38 \%$ y $24 \%$, respectivamente. Mientras que en Smicridea se halló MOPF, MOPG y TPV, con $38 \%$, $34 \%$ y $20 \%$. El Análisis Discriminante de Hotelling mostró diferencias significativas en la dieta de estos géneros $(\mathrm{F}=8.8298, \mathrm{p}<0.05)$.

En el contenido estomacal de Smicridea se identificaron varias microalgas, dentro de las cuales se destacan diatomeas céntricas como Melosira (Melosirales, Fig. 5A), y diatomeas pennadas del género Synedra, Pinnularia y Navicula (Fig. 5B). Así mismo, también se observó tejidos de plantas vasculares (Fig. 5C), MOPF (Fig. 5D), MOPG (Fig. 5E) y restos de animales pertenecientes a larvas de Simulium (Fig. 5F). Por otra parte, en los intestinos de Leptonema se encontró diatomeas céntricas del género Melosira (Fig. 5G), tejidos de plantas vasculares (Fig. 5H), MOPF (Fig. 5I), MOPG (Fig. 5J) y restos de animales, que corresponden a una mandíbula de Chimarra (Trichoptera: Philopotamidae) (Fig. 5K) y una parte del abdomen de un efemeróptero (Fig. 5L).

\section{DISCUSIÓN}

En general, la estructura morfológica de las piezas bucales de Smicridea fue similar a las descripciones presentadas por Duque (2009), con excepción de larvas de Smicridea (Rhyacophylax) appendiculata, quien presentó diferencias notorias en la coloración y forma de las mandíbulas (seis dientes). Las larvas

CUADRO 2

Contenido estomacal en porcentaje y tallas promedio para Leptonema y Smicridea

TABLE 2

Stomach contents percentage and average size for Leptonema and Smicridea

\begin{tabular}{rcccccccc} 
Género & No ind. analizados & LT $(\mathrm{mm}) \pm \mathrm{DE}$ & RA & TPV & MCR & HN & MOPG & MOPF \\
Leptonema & 8 & $16.49 \pm 1.59$ & 1.06 & 24.05 & 2.93 & 0.66 & 38.35 & 32.93 \\
Smicridea & 7 & $15.71 \pm 1.22$ & 1.19 & 20.67 & 4.67 & 0.68 & 34.05 & 38.78 \\
\hline
\end{tabular}

*Abreviaturas: $\mathrm{LT}=$ longitud total expresada en mm con una desviación estándar $(\mathrm{DE}), \mathrm{RA}=$ restos animales, $\mathrm{TPV}=$ tejido de plantas vasculares, $\mathrm{MCR}=$ microalgas, $\mathrm{HN}=$ hongos, $\mathrm{MOPG}=$ materia orgánica particulada gruesa y $\mathrm{MOPF}=\mathrm{materia}$ orgánica particulada fina. 


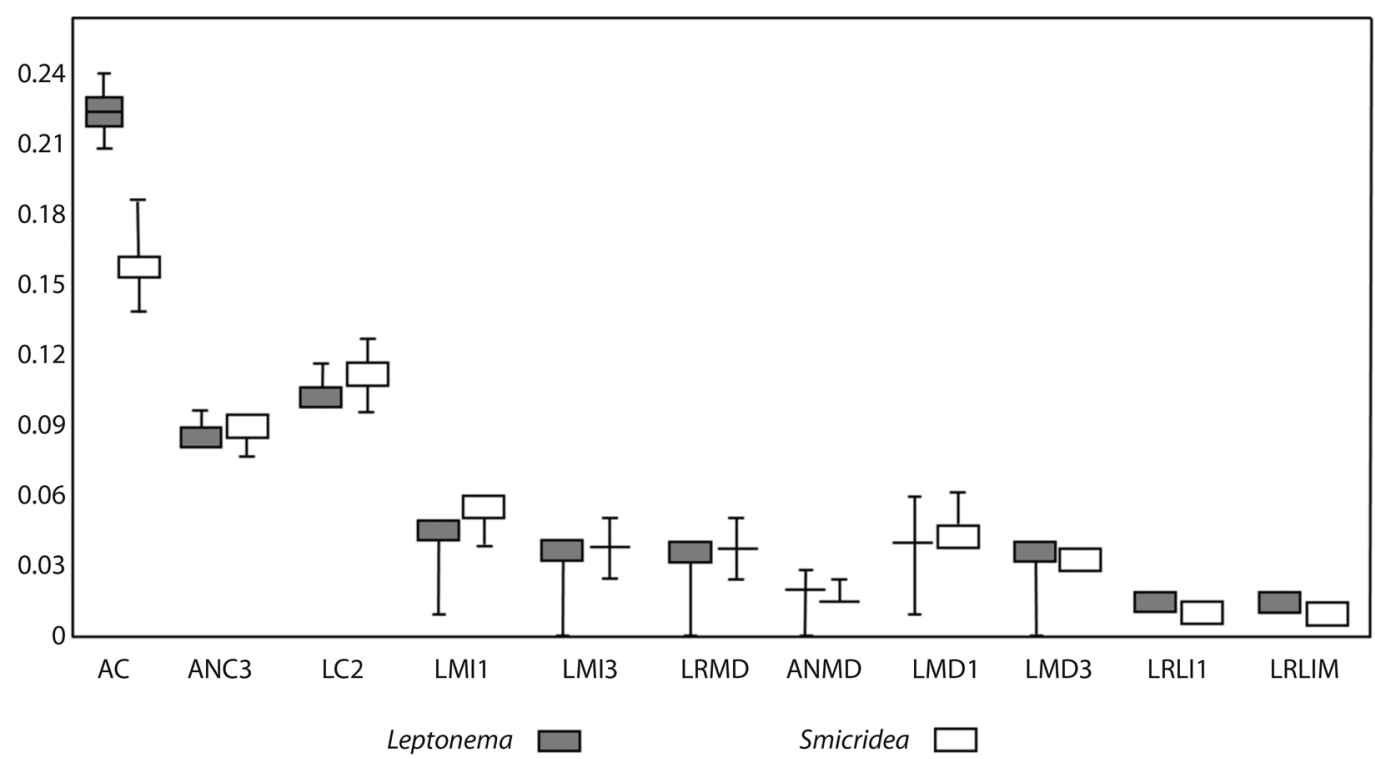

Fig. 4. Diagrama de cajas y bigotes donde se compara las variables morfológicas entre los géneros Leptonema y Smicridea: AC: área de la cabeza, ANC3: ancho de la cabeza 3, LC2: largo de la cabeza 2, LMI1: largo de la mandíbula izquierda 1, LMI3: largo de la mandíbula izquierda 3, LRMD: largo de la mandíbula derecha, ANMD: ancho de la mandíbula derecha, LMD1: lado de la mandíbula derecha 1, LMD3: lado de la mandíbula derecha 3, LRLI1: largo del labrum inferior 1, LRLIM: largo del labrum inferior medio.

Fig. 4. Box and whisker diagram where different morphological variables compared between Leptonema and Smicridea genera. AC: head area, ANC3: head width 3, LC2: long head 2 LMI1: long the left mandible 1, LMI3: along the left mandible 3, LRMD: along the right mandible, ANMD: width of the right mandible, LMD1: right side of the mandible 1, LMD3: right side of the mandible 3, LRLI1: long the lower labrum 1, LRLIM: long the middle lower labrum.

de este estudio, presentaron en la mandíbula izquierda ocho dientes y en la derecha seis. Por otro lado, las descripciones de Leptonema en este estudio, coinciden en su mayoría con las realizadas por Muñoz (1999) en Costa Rica y Nessimian y Dumas (2010) en Brasil, quienes caracterizaron la cabeza, el labrum, el complejo hipofaringeal y las mandíbulas. No obstante, en este estudio, la mandíbula izquierda presentó un penacho de setas en la región dorsomolar en vista dorsal y ocho dientes, a diferencia de las evaluadas en Brasil y Costa Rica, que carecen de este penacho y presentan siete dientes en la mandíbula izquierda.

Spänhoff, Schulte, Alecke, Kaschek y Meyer (2003) revisaron la morfología del aparato bucal y los hábitos alimentarios de Lype phaeopa (Trichoptera, Psychomyiidae) y se evidenció diferencias a este estudio en la esclerotización del labrum, que no se encontró en
Leptonema y Smicridea. Esto se puede explicar a partir de que L. phaeopa raspa las capas superficiales de madera, para lo cual necesita estructuras fuertes. En relación al estudio morfométrico del aparato bucal, no se encontró trabajos relacionados para ninguno de los géneros Leptonema y Smicridea. Solo en una descripción taxonómica hecha por Springer (2010), en donde se hace referencia a que Smicridea presenta una cabeza cuadrada y Leptonema una cabeza alargada (que se hace más ancha en la región posterior), coincide con los resultados de la morfometría de los organismos evaluados en el estudio.

Por otra parte Bournaud, Tachet y Perrin (1982) implementaron dos criterios de identificación: la descripción morfológica y las características de coloración, con el fin de registrar nuevas especies de Hydropsychidae, esto debido a la complejidad que se tenía 

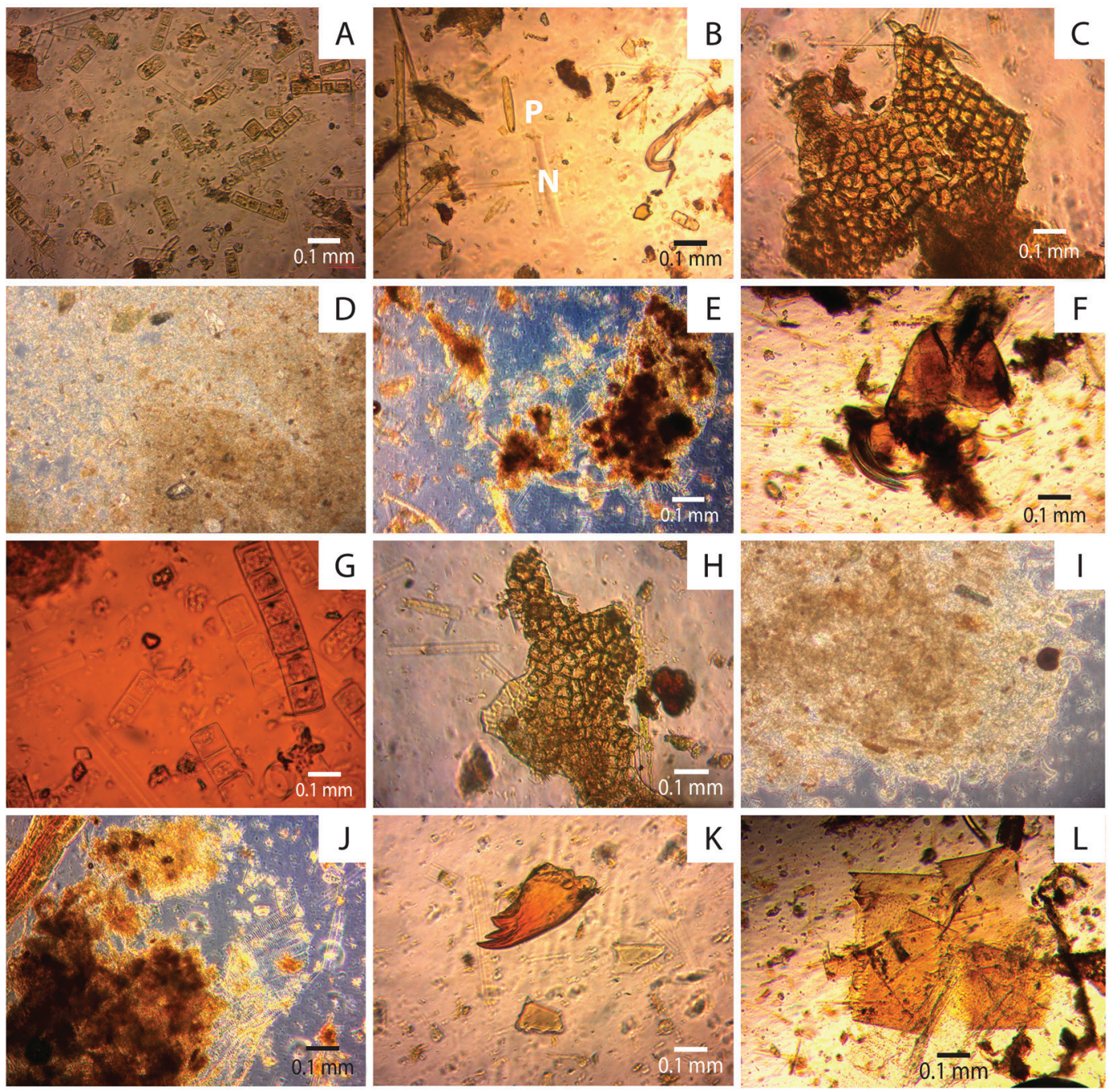

Fig. 5. Smicridea: A) Microalga (Melosira sp.) (40x). B) Microalga (Synedra sp. (S); Pinnularia sp. (P) y Navicula sp. (N) (20x). C) tejido de plantas vasculares (40x). D) materia orgánica particulada fina (MOPF) (20x). E) materia orgánica particulada gruesa (MOPG) (20X). F) restos animales (Simulium) (40x). Ítems encontrados en los intestino de Leptonema: G) Microalga (Melosira sp.) (40x). H) tejido de plantas vasculares (40x). I) materia orgánica particulada fina (MOPF) (20x). J) materia orgánica particulada gruesa (MOPG) (20X). K) mandíbula de Chimarra y. L) abdomen de un efemeróptero (40x). Fig. 5. Smicridea. A) microalgae (Melosira sp.) (40x). B) microalgae [Synedra sp. (S); Pinnularia sp. (P) and Navicula sp. (N)] (20x). C) vascular plant tissue (40x). D) fine particulate organic matter (MOPF) (20x). E) coarse particulate organic matter (MOPG) (20X). F) animal remains (Simulium) (40x). Items found in the gut of Leptonema: G) microalgae (Melosira sp.) $(40 \mathrm{x})$. H) vascular plant tissue $(40 \mathrm{x})$. I) fine particulate organic matter (MOPF) (20x). J) coarse particulate organic matter (MOPG) (20X). K) mandible Chimarra. L) Abdomen of an ephemeroptera (40x).

para su determinación. Algunas características morfológicas tenidas en cuentas fueron: forma del borde delantero de la apótoma frontoclipeal, forma de apótema, forma de la cabeza (relación entre anchura y longitud) y forma submentón. A partir de esto, lograron identificar siete especies de Hydropsychidae, la implementación de las descripciones morfológicas y las características de coloración contrastan con las descripciones de este estudio, donde se tuvieron en cuenta otras estructuras como mandíbulas, labrum y complejo hipofaringeal. 
Para Toro, Manriquez y Suazo (2010), la morfología en sus inicios se centró en las descripciones de estructuras observadas como sus dimensiones y sus formas. La estructura podía ser descrita minuciosamente, pero no podría ser analizada cuantitativamente, en el actual estudio se describieron las estructuras cefálicas cualitativamente, así como también cuantitativamente. La morfometría es una importante herramienta para la evaluación objetiva de las variaciones de forma.

Con relación a la dieta, los mayores ítems consumidos fueron MOPF y MOPG para ambos géneros, que representaron las principales fuentes energéticas (Granados, 2013; Guzmán-Soto \& Tamaris-Turizo, 2014). Gran parte de la MOPF se genera por la descomposición de la MOPG como lo expresa Allan y Castillo (2007). Este constituye un elemento permanente y debido a su alta disponibilidad en los ecosistemas lóticos explicaría su presencia con frecuencia en los contenidos estomacales de los géneros estudiados (Tomanova et al., 2006), como se presenta en el actual estudio con los géneros Leptonema y Smicridea. Los organismos evaluados de ambos géneros evidenciaron una preferencia de por lo menos tres ítems alimentarios, en función de las altas proporciones promedio observadas implicando que ninguno de los individuos presentó una dieta exclusiva, lo que concuerda con diferentes autores al respecto, quienes consideran a los insectos acuáticos como oportunistas al presentar una heterogeneidad en los ítems alimentarios (Motta \& Uieda, 2004; Merritt et al., 2008). Reynaga (2009) y Rodríguez et al. (2014) manifiestan que el género Smicridea parecería tener un rol depredador de invertebrados procedentes de la deriva, lo que puede explicar la presencia de restos de larvas de Simulium en sus intestinos. Smicridea y Leptonema habitan en refugios creados por ellos mismos, y se alimentan de las partículas que adhieren en la red que forman, por lo cual no se podrían considerar como depredadores. En efecto Ramírez y Gutiérrez-Fonseca (2014), los clasifican como filtradores.
En el análisis discriminante de Hotelling aplicado a la composición de las dieta mostró diferencias entre ambos géneros, a pesar de que consumen los mismos recursos como la MOPG, MOPF y TPV. Lo que sugiere que las proporciones del perfil dietario difieren entre ambos. Para Tomanova et al. (2006) pueden encontrarse diferencias en las proporciones de ingestas las cuales podrían estar vinculadas a una alta o baja disponibilidad del recurso en el medio. Este criterio puede estar asociado a que las larvas de los géneros Leptonema y Smicridea construyen redes finas en las corrientes de agua con materiales orgánicos y minerales con las que filtrar el agua (Merritt et al., 2008) o bien pueden ubicarse en las grietas de las piedras (Duque, 2009). Dichos estudios han permitido reconocer aspectos de la biología de estos organismos. En todo caso, es necesario evaluar la disponibilidad de los recursos y las diferencias morfológicas de las piezas cefálicas de estos géneros para poder llegar a dichas afirmaciones.

Los escasos estudios de morfometría y análisis de contenido estomacal que se han realizado para Leptonema y Smicridea en América no incluyen a ambos géneros simultáneamente, por el contrario la generalidad corresponde a evaluaciones de manera individual. A pesar de que son géneros diferentes en la morfología de sus pizas bucales, en vista general son muy similares. En este estudio se hizo una comparación entre ambos géneros que sugieren diferencias en la eficiencia de reciclaje de la materia como recurso disponible en el ambiente, cuyo tema puede ser planteado en futuros estudios que aborden un nivel taxonómico de especie y observaciones de los organismos vivos con diferencias en la disponibilidad de recursos alimentarios bajo condiciones controladas. Por otra parte, se puede tener en cuenta las piezas bucales a la hora de identificar ambos géneros.

\section{AGRADECIMIENTOS}

Los autores agradecen el apoyo brindado por el Laboratorio de Entomología de la Universidad del Magdalena. A Tania Karelys 
Franco por su valiosa asesoría en la toma de fotos. A Yesely Hurtado por el apoyo en campo.

\section{RESUMEN}

Los géneros Leptomea y Smicridea pertenecen a la familia Hidropsychidae y constituyen uno de los grupos más importantes en la dinámica de nutrientes en los ecosistemas acuáticos tropicales, cuya diversidad de microhabitats es aprovechada por las larvas de estos géneros. Sin embargo, se dispone de escasa información que describa y compare las piezas bucales entre ambos géneros y su posible relación con la dieta. El objetivo de este trabajo fue describir la morfología de las piezas bucales y los hábitos alimentarios de ambos géneros en la cuenca media del río Gaira (Sierra Nevada de Santa Marta, Colombia). Las muestras se recolectaron mensualmente con una red Surber entre octubre y diciembre 2014 y mayo 2015. Se utilizó el Software Zen Blue $\subset$ para las estimaciones morfométricas de las piezas cefálicas, utilizando 15 larvas por género. Las medidas se tomaron sobre fotografías obtenidas de una cámara AxioCam ERc5s ajustada a un estereoscopio Nikon SMZ 745T. Para la descripción detallada de las piezas cefálicas, se usó un microscopio-estereoscopio Leica M205A. Se evaluaron los hábitos alimentarios de 15 individuos en total, mediante el análisis de contenido estomacal. Se aplicó un análisis discriminante de Hotelling a las medidas morfométricas. Se encontraron diferencias significativas en el área de la cabeza (AC), el ancho de la cabeza (ANC3), largo de la cabeza (LC2) y largo de la mandíbula izquierda (LMI1). En la descripción morfológica se hallaron diferencias en: cabeza, labrum y submentón. Mediante el análisis del contenido estomacal se realizaron los perfiles dietarios para ambos géneros y se detectó que los ítems con mayor porcentaje para Leptonema fueron MOPG (38 \%) y MOPF (32\%) y para Smicridea, MOPF (38\%) y MOPG (34\%), con diferencias significativas $(\mathrm{F}=8.8298, \mathrm{p}<0.05)$. Este estudio indica que los individuos evaluados consumen una amplia variedad de recursos y la diferencia entre la dieta de ambos géneros puede estar relacionada con las diferencias morfológicas detectadas.

Palabras clave: insectos acuáticos, morfometría, contenido estomacal, aparato bucal, ítem alimentario.

\section{REFERENCIAS}

Allan, J., \& Castillo, M. (2007). Stream Ecology. Structure and function of running waters. Dordrecht: Springer.

Angrisano, E., \& Korob, P. G. (2001). Trichoptera. In H. R. Fernández \& E. Domínguez (Eds.), Guía para la Determinación de los Artrópodos Bentónicos Sudamericanos (pp. 55-92). Tucumán, Argentina: Editorial Universitaria de Tucumán.
Angrisano, E., \& Sganga, J. (2009). Trichoptera. En E. E. Domínguez \& H. R. Fernández (Eds.), Macroinvertebrados Bentónicos Sudamericanos. Sistemática y Biología (pp. 255-308). Tucumán, Argentina: Fundación Miguel Lillo.

Bournaud, M., Tachet, H., \& Perrin, J. (1982). Les Hydropsychidae (Trichoptera) Du Haut-Rhone Entre Geneve Et Lyon. Annales de Limnologie - International Journal of Limnology, 18(1), 61-80. Recuperado de http://www.limnology-journal.org/articles/limn/ pdf/1982/01/limn1982181p61.pdf

Chará, A., Chará, J., Zúñiga, M., Pearson, R., \& Boyero, L. (2012). Diets of leaf litter-associated invertebrates in three tropical streams. Annales de Limnologie - International Journal of Limnology, 48(2), 139-144. doi: 10.1051/limn/2012013

Darrigran, G., Vilches, A., Legarralde, T., \& Damborenea, C. (2007). Guía para el estudio de macroinvertebrados: Métodos de colecta y técnicas de fijación. Buenos Aires, Argentina: ProBiota-FCN \& M-UNLP.

Duque, J. (2009). Taxonomia do gênero Smicridea McLachlan (Trichoptera: Hydropsychidae) de três estados da Amazônia (Tesis de Mestre). Universidade do Amazonas, Manaus, Amazonas.

Frayter, V., Pabón, R., \& Valero, O. (2000). Plan de manejo integral de la cuenca hidrográfica del río Gaira (Tesis de pregrado). Universidad del Magdalena, Santa Marta, Colombia.

Granados, C. (2013). Análisis de la dieta de los macroinvertebrados bentónicos en un gradiente altitudinal de la cuenca del río Gaira (Sierra Nevada de Santa Marta - Colombia) (Tesis de Maestría). Universidad del Zulia, Zulia, Venezuela.

Guerrero, R., \& Sarmiento, C. (2010). Distribución altitudinal de hormigas (Hymenoptera, Formicidae) en la vertiente noroccidental de la Sierra Nevada de Santa Marta (Colombia). Acta Zoológica Mexicana, 26(2), 279-302. Recuperado de http://www.scielo.org.mx/ pdf/azm/v26n2/v26n2a3.pdf

Guerrero, F., Manjarrés, A., \& Núñez, N. (2003). Los macroinvertebrados bentónicos de pozo azul (cuenca del río Gaira, Colombia) y su relación con la calidad del agua. Acta Biológica Colombiana, 8(2), 43-55.

Guzmán-Soto, C., \& Tamarís-Turizo, C. (2014). Hábitos alimentarios de individuos inmaduros de Ephemeroptera, Plecoptera y Trichoptera en la parte media de un río tropical de montaña. Revista de Biología Tropical, 62(2), 169-178.

Huamantinco, A., \& Nessimian, J. (2000). Variation and life strategies of the Trichoptera (Insecta) larvae community in a first order tributary of the Paquequer river, southeastern Brazil. Brazilian Journal of Biology, 60(1), 73-82. doi: http://dx.doi.org/10.1590/ S0034-71082000000100010 
Merritt, R. M., Cummnins, K. W., \& Berg, M. B. (2008). An introduction to the aquatic invertebrates of North America. Dubuque, Estados Unidos: Kendall Hunt.

Motta, R., \& Uieda, V. (2004). Diet and trophic groups of an aquatic insect community in a tropical stream. Brazilian Journal of Biology, 64(4), 809-817. doi: 10.1590/S1519-69842004000500010

Muñoz, F. (1999). El género Leptonema (Trichoptera: Hydropsychidae) en Costa Rica, con la descripción de una nueva especie. Revista de Biología Tropical, 47(4), 959-1006

Nessimian, J., \& Dumas, L. (2010). Description of the immature stages of Leptonema tridens (Insecta: Trichoptera: Hydropsychidae) from southeastern Brazil with notes on its biology. Zoologia, 27(3), 465-471. doi: 10.1590/S1984-46702010000300021

Pes, A. (2005). Taxonomia, estrutura e riqueza das assembléias de larvas e pupas de Trichoptera (Insecta), em igarapés na Amazônia Central (Tese de doutorado). Universidade do Amazonas, Manaus, Amazonas. En J. Duque. 2009. Taxonomia do gênero Smicridea McLachlan (Trichoptera: Hydropsychidae) de três estados da Amazônia (Tesis de Mestre). Universidade do Amazonas, Manaus, Amazonas.

Posada, J., \& Roldán, G. (2003). Illustrated key for the larvae of Trichoptera in the northwest of Colombia. Caldasia, 25(1), 169-192. Recuperado de http:// www.revistas.unal.edu.co/index.php/cal/article/ viewFile/39407/41298

R Development Core Team. (2012). R: A language and environment for statistical computing. R Foundation for Statistical Computing. Viena, Austria. Recuperado de http://www.R-project.org/

Ramírez, A., \& Gutiérrez-Fonseca, P. E. (2014). Functional feeding groups of aquatic insect families in Latin America: a critical analysis and review of existing literature. Revista de Biología Tropical, 62(Suppl. 2), 155-167.

Reynaga, M. C. (2009). Hábitos alimentarios de larvas de Trichoptera (Insecta) de una cuenca subtropical. Ecología Austral, 19(3), 207-214. Recuperado de http:// www.scielo.org.ar/pdf/ecoaus/v19n3/v19n3a04.pdf

Rodríguez, V., Gracia, V., \& Peña, B. (2014). Familias y géneros de larvas de Trichoptera en los ríos de la provincia de Veraguas y su clasificación trófica en grupos alimenticios funcionales. Tecnociencia, 16(2), 33-53.

Springer, M. (2010). Capítulo 7: Trichoptera. Revista de Biología Tropical, 58(4), 151-198. Recuperado de http://www.scielo.sa.cr/pdf/rbt/v58s4/a07v58s4.pdf

Springer, M., Serrano, L., \& Zepeda, J. (2010). Guía ilustrada para el estudio ecológico y taxonómico de los insectos acuáticos inmaduros del Orden Trichoptera. En J. M. Sermeño Chicas (Ed.), Formulación de una guía metodológica estandarizada para determinar la calidad ambiental de las aguas de los ríos de El Salvador, utilizando insectos acuáticos (pp. 6-48). San Salvador, El Salvador: Universitaria UES.

Spänhoff, B., Schulte, U., Alecke, C., Kaschek, N., \& Meyer, E. (2003). Mouthparts, gut contents, and retreatconstruction by the wood-dwelling larvae of Lype phaeopa (Trichoptera, Psychomyiidae). European Journal of Entomology, 100(4), 563-570. doi: http://dx.doi.org/10.14411/eje.2003.085

Tamaris-Turizo, C. E., Rodríguez-Barrios, J., \& OspinaTorres, R. (2013). Deriva de macroinvertebrados acuáticos a lo largo del río Gaira, vertiente noroccidental de la Sierra Nevada de Santa Marta, Colombia. Caldasia, 35(1), 149-163.

Tomanova, S., Goitia, E., \& Helesic, J. (2006). Trophic levels and functional feeding groups of macroinvertebrates in neotropical streams. Hydrobiologia, 556, 251-264. doi: 10.1007/s10750-005-1255-5

Toro, M., Manriquez, G., \& Suazo, I. (2010). Morfometría geométrica y el estudio de las formas biológicas: de la morfología descriptiva a la morfología cuantitativa. International Journal of Morphology, 28(4), 977-990.

Valladolid, M., Martínez, J., \& Arauzo, M. (2007). Los Hydropsychidae (Insecta: Trichoptera) del río Oja (La Rioja, España). Limnetica, 26(1), 199-208.

Vannote, R. L., Minshall, G. W., Cummins, K. W., Sedell, J. R., \& Cushing, C. E. (1980). The River Continuum Concept. Canadian Journal of Fisheries and Aquatic Sciences, 37, 130-137.

Wiggins, G. B. (2004). Caddisflies. The Underwater Architects. Toronto, Canada: University of Toronto Press. 